11 Abstract

12

\title{
Nanometric axial localization of single fluorescent molecules with modulated excitation.
}

\author{
Pierre Jouchet ${ }^{1}$, Clément Cabriel ${ }^{1}$, Nicolas Bourg ${ }^{1}$, Marion Bardou ${ }^{1}$, Christian Poüs ${ }^{2}$, Emmanuel Fort ${ }^{3}$, \\ Sandrine Lévêque-Fort ${ }^{1{ }^{1 *}}$ \\ ${ }^{1}$ Université Paris Saclay, CNRS, Institut des Sciences Moléculaires d’Orsay, 91405, Orsay, France \\ 2 Université Paris Saclay, INSERM UMR-S 119, Châtenay-Malabry, France \\ ${ }^{3}$ Institut Langevin, ESPCI Paris, CNRS, PSL University, Paris, France \\ Corresponding author: sandrine.leveque-fort@u-psud.fr
}

Distance measurements are commonly performed by a phase detection based on a lock-in strategy. Superresolution fluorescence microscopy is still striving to perform axial localization but through entirely different strategies. Here we show that an illumination modulation approach can achieve nanometric axial localization precision without compromising on the acquisition time, emitter density or lateral localization precision. The excitation pattern is obtained by shifting tilted interference fringes. The molecular localizations are performed by measuring the relative phase between each fluorophore response and the reference modulated excitation pattern. We designed a fast demodulation scheme compatible with the short emission duration of single emitters. This modulated localization (ModLoc) microscopy offers a typical axial localization precision of $6.8 \mathrm{~nm}$ over the entire field of view and the axial capture range. Furthermore, the interfering pattern being robust to optical aberrations, a nearly uniform axial localization precision enables imaging biological samples up to several micrometers in depth. 
Fluorescence microscopy is currently the most widespread technique for cell imaging. It has now achieved the nanometer scale with the advent of super-resolution techniques such as single molecule localization microscopy $(\mathrm{SMLM})^{1-4}$. However, axial localization is still an ongoing central issue ${ }^{5}$. The symmetry of the microscopes and their optical optimization favor the transverse direction compared to the axial one. Several strategies have been developed in SMLM based on point spread function (PSF) handling like multifocused techniques ${ }^{6-8}$, PSF engineering ${ }^{9-12}$ or supercritical angle fluorescence ${ }^{13-15}$. Alternative techniques retrieve axial localization based on the coherence of a single emitter fluorescence, like interferometric PALM (iPALM) ${ }^{16,17}, 4 \mathrm{Pi}$ single marker switching nanoscopy (4Pi-SMSN) $)^{18,19}$ or Self-Interference $\left(\right.$ SelFi) ${ }^{20}$. The axial localization is often performed at the expense of the lateral one, reducing the working depth ${ }^{12,21}$ or the field of view ${ }^{20}$. Most techniques optimized in the focal plane quickly degrade with defocus. In addition, their performances are hindered by the optical aberrations within the sample beyond a few micron depths unless adaptive optics ${ }^{19,22}$ or more robust fluorescence self-interference strategies ${ }^{20}$ are used.

In the case for coherent light, interferometry offers unmatched sensitivity for distance measurements by detecting the relative phase between two electromagnetic wave ${ }^{23}$. This phase is often obtained by modulation using a standard lock-in detection. This strategy which has been used in coherent microscopy configurations to obtain nanometric axial localization ${ }^{24-26}$, cannot be implemented directly for the noncoherent fluorescence emission. However, localization by lock-in detection can be adapted to amplitude modulation like in Light Detection and Ranging (LIDAR) or Time of Flight (TOF) cameras $^{27}$. Since the response of the fluorophores is linear in intensity (providing sufficiently low excitation), it is possible to retrieve the phase of the fluorescence signal emitted by a single emitter with a lock-in detection using time-modulated structured illumination as a reference. This idea has been used for the transverse direction in the early days of single emitter tracking to probe myosin $V$ stepping mechanism ${ }^{28}$ with gold nanobeads ${ }^{29}$. The illumination is obtained by a shifting interference pattern and the detection performed by a fast mono-detector. Recently, this strategy has been revived to single molecule transverse localization for SMLM ${ }^{30,31}$. However, the lock-in detection using a slow camera is performed sequentially and excludes a significant proportion of the emitters due to their limited emission time. 


$$
Z=\frac{\lambda_{\mathrm{exc}} \phi}{4 \pi n \sin \left(\frac{\alpha}{2}\right) \sin (\theta)}-\frac{x}{\tan (\theta)}
$$

$72 \sigma_{\text {ModLoc }}=\frac{1}{\sin \theta \sqrt{N}} \sqrt{\frac{\lambda_{\text {exc }}{ }^{2}}{16 \pi^{2} n^{2} \sin ^{2}\left(\frac{\alpha}{2}\right)}+\sigma_{\mathrm{PSF}}^{2} \cos ^{2} \theta}$ with $N$ the number of detected photons and $\sigma_{\mathrm{PSF}}$ the PSF standard deviation (Supplementary Note 1 ). 

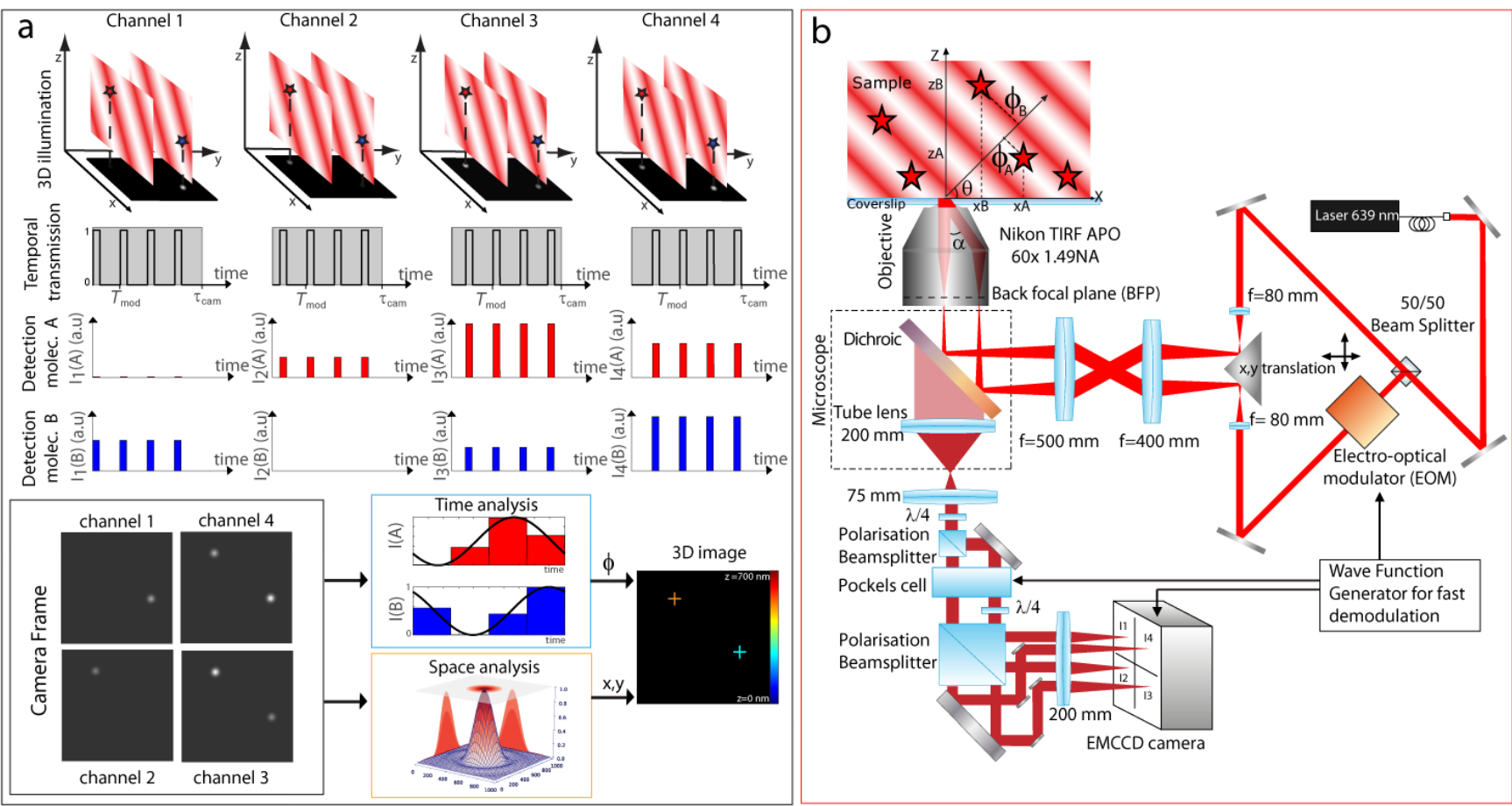

Figure 1 / Modulated localization (ModLoc) principle and experimental implementation. a, Fast demodulation is based on 4 time channels: for each channel the xz tilted illumination pattern is in quadrature relative to one another. The 4 time channels have complementary transmission lasting a quarter of a modulation period $T_{\text {mod }}$, repeated during multiple modulation cycles within the total acquisition time $\tau_{\text {cam. }}$. Illustration of the detected intensities in the four different channels for two molecules ( $A$ and $B$ ) is represented. These 4 channels are acquired simultaneously on the camera. A centroid fitting of the PSF gives access to the lateral $x, y$ localization for each molecule. The phase $\phi$ of their modulated emission is retrieved from the relative PSF intensity in each channel. The combination of the two information leads to a 3D image. $\boldsymbol{b}$, Implementation of ModLoc microscopy: The tilted illumination pattern $\left(\lambda_{\text {exc }}=\right.$ $639 \mathrm{~nm}$ ) is obtained by the interference of two beams focused in the back focal plane of the 1.49 NA objective with tunable off-axis distance to control the interference period and the fringe angle $\theta$ in the sample. The time modulation frequency is performed by shifting the relative phase between the two excitation beams using an electro-optic modulator (EOM). The lock-in demodulation is performed thanks to a dedicated detection module based on polarization sorting: a $\lambda / 4$ plate, followed by a polarization beamsplitter, a Pockels cell, a $\lambda / 4$ plate in one of the path shift the polarization state to obtain 4 complementary channel after a last polarization beamsplitter. The initial fluorescence image is steered into a set of 4 detection channels with complementary time varying transmission performed by a Pockels cell and synchronized with the EOM. These 4 channels are imaged simultaneously on the EMCCD camera. The modulation/demodulation frequency, typically between $600 \mathrm{~Hz}$ to $1200 \mathrm{~Hz}$, is chosen to be compatible with the short $\mathrm{ON}$-time of the emitters typically averaging over 30 to 60 modulation cycles.

For the high Numerical Aperture (NA) objectives used in SMLM, the axial modulation period $\Lambda_{\mathrm{z}}$ is of the order of $\lambda_{\text {exc }}$. The ultimate limit for a single objective excitation configuration is given by $\theta=\alpha / 2=45^{\circ}$ and results in a quasi-isoprecision of $\sigma_{\text {ModLoc }} \approx 1.1 \sigma_{x}, \sigma_{x}$ being the standard deviation in the lateral direction $x$. In this configuration however, $\Lambda_{\mathrm{z}}$ being smaller than the objective capture range, unwrapping strategies are needed to extract the axial information (Supplementary Fig.1). In the following to avoid phase wrapping, $\Lambda_{\mathrm{z}}$ is set to obtain a unique solution between the measured phase and the axial emitter 
position. This preserves a straightforward observation over the whole depth of focus but reduces the axial localization compared to the optimal configuration.

103 The modulation frequency must be compatible with the limited ON-time emission of the molecules. The 104 emitters have an ON-time with a Poissonian statistic which depends on the illumination intensity. To fulfill 105 time sparsity and localize most of the molecules in a single shot, $\tau_{\mathrm{ON}}$ must satisfy $\tau_{\mathrm{ON}}<\tau_{\text {cam }}$, $\tau_{\text {cam }}$ being 106 the minimum exposure time of the camera to optimize the acquisition time. For example, we measured 107 for our experiments $\tau_{\mathrm{ON}}=15 \mathrm{~ms}$ for AF647 observed in (d)STORM (Supplementary Fig. 2). The modulation period $T_{\text {mod }}=2 \pi / \Omega$ must satisfy $T_{\text {mod }} \ll \tau_{\mathrm{ON}}$ to retrieve the phase for the most of the emitters (Supplementary Fig. 3). Typically, frequencies satisfying an average molecular ON-time lasting at least a few tens of modulation cycles are optimal (Supplementary Fig. 4) which hindered the sequential

111 acquisition strategy. Figure 1a shows the principle of the fast lock-in detection strategy which must be 112 developed to perform the demodulation.

113 The lock-in detection is performed with 4 time channels with complementary transmissions lasting a 114 quarter of a modulation period $T_{\text {mod }}$ (Fig. 1a). Alternative transmission functions are possible, but they 115 should be complementary to avoid any photon loss. Each channel is associated to an illumination pattern 116 in quadrature relative to one another and imaged into a specific subarray of the camera. Thanks to sparsity,

117 the fluorescence intensity $I_{q}$ transmitted into channel $q$ by each fluorophore after integration by the 118 camera can be measured and the phase deduced by $\phi=\frac{1}{2 \pi}$ atan $\left(\frac{I_{4}-I_{2}}{I_{3}-I_{1}}\right)$. With the lateral position obtained 119 from centroid fitting of the PSF, the axial position of the fluorophore can be deduced using eq (1).

\section{Experimental setup}

122 Figure $1 \mathrm{~b}$ shows the experimental implementation of the ModLoc microscope (Methods and 123 Supplementary Fig. 1-10 for details). The tilted illumination pattern is obtained by the interference of the 124 two parallel beams focused in the back focal plane of the high NA objective. The $639 \mathrm{~nm}$ laser beam is 125 coupled through a polarizing fiber and divided in two symmetrical beam paths. Their off-axis distance can 
be adjusted using a knife-edge mirror mounted on an $x, y$ translation or independently using mirrors placed after (not shown in Fig.1b). The interference fringe pattern is shifted with an electro-optic modulator (EOM) placed in one of the beams to tune their relative phase. The fast lock-in demodulation is performed with a specific scheme based on polarization sorting. The initial fluorescence image is sorted into 4 detection channels with complementary time varying transmission synchronized with the excitation modulation (Supplementary Note 2 and Supplementary Fig. 5). The initial image is first split in two complementary orthogonal polarization channels. The modulated transmission is obtained using a Pockels cell in each path and splitting them into two complementary channels using a polarizing cube. To shift a modulation in quadrature, a quarter waveplate is introduced in one of the path after the Pockels cell. Standard registration code is used to associate the four intensity values and positions (Supplementary Fig.6). Typically, the modulation frequency is set between $600 \mathrm{~Hz}$ and $1200 \mathrm{~Hz}$ for an acquisition rate of the camera of $20 \mathrm{~Hz} .30$ to 60 modulation cycles are observed for each frame, allowing the detection of most emitters even with short ON-time. As for classical SMLM imaging, 10000 to 20000 images are typically acquired to reconstruct the final super-resolved image. Standard Direct Cross Correlation ${ }^{32}$ algorithm are applied to retrieve drifts in all directions (Methods).

To avoid phase wrapping and optimize axial localization, $\Lambda_{z}$ is set to approximately $1 \mu \mathrm{m}$ (Supplementary Fig. 1 and Notes 1 ) is obtained with $\alpha=42^{\circ}$ and $\theta=44^{\circ}$. This configuration with expected axial standard deviation $\sigma_{\text {ModLoc }} \approx 2.5 \sigma_{x}$ is used to image from the coverslip to several tens of microns in depth without unwrapping (Supplementary Note 3 and Supplementary Fig. 1, 7, 8 , 9 and 10 ).

\section{Results}

We first characterized ModLoc axial and lateral localization precisions at various depths. To evaluate the intrinsic precision, we performed repeated localizations on single emitters (Atto647N) with DNA-PAINT labelling whose long activation times ( $>100 \mathrm{~ms}$ ) allow a repeated localization over consecutive frames (see Methods). Fig. 2a shows the very good agreement between the experimental data and the theoretical limits (Cramèr Rao Lower Bound, CRLB) as a function of detected photons (Supplementary Fig. 11 and 12, 

$\pm 0.4 \mathrm{~nm}$ and $3.1 \pm 0.2 \mathrm{~nm}$ respectively.
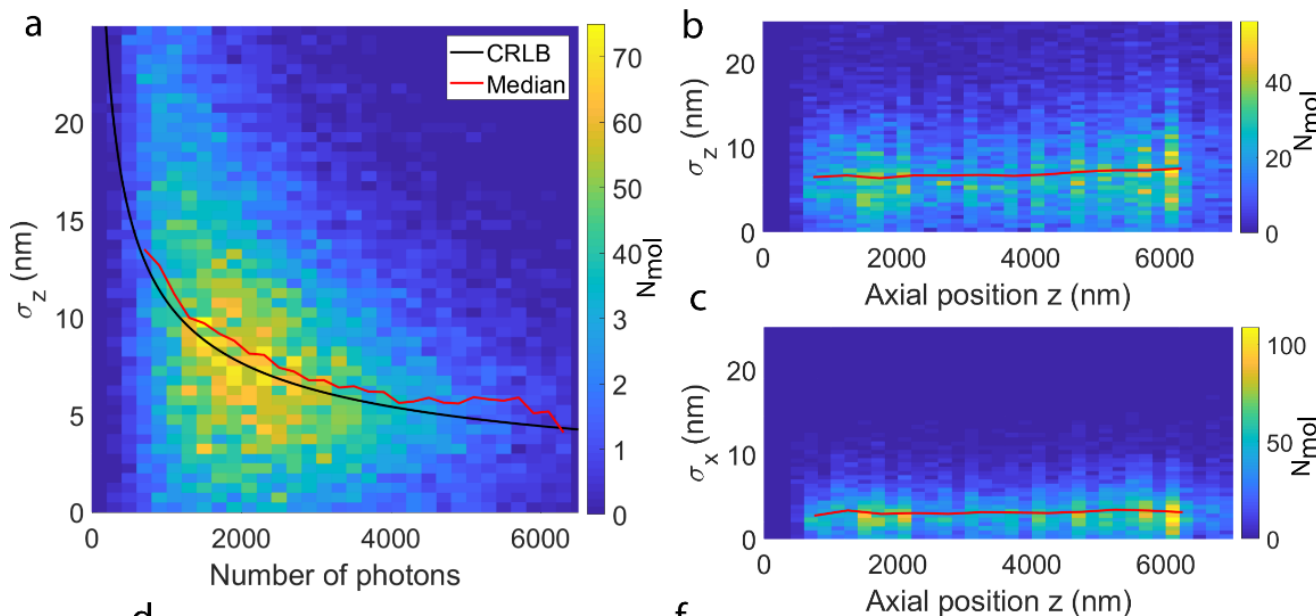

d

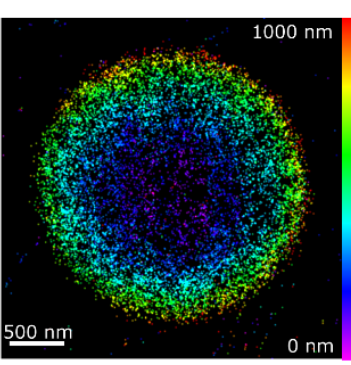

e
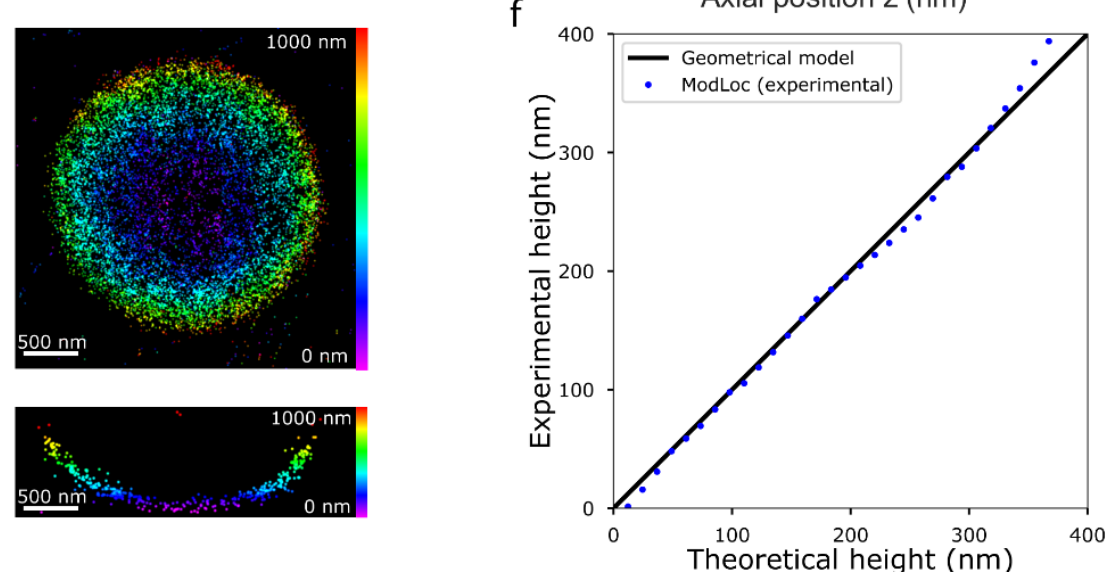

Figure 2 I Performances of ModLoc. The experimental localization precisions are obtained from the standard deviation of the localization distribution for single molecular emitters detected on several successive frames (ATTO647N in DNA-PAINT).a, Histogram of the axial localization precision of ModLoc as a function of the number of detected photons. The red curve is the experimental median and the black curve is the Cramèr-Rao lower bounds (CRLB). $\boldsymbol{b}$ and $\boldsymbol{c}$ Histogram of the localization precision in the axial and lateral direction respectively acquired at various depths up to $7 \mu \mathrm{m}$. Their associated median values (red lines). The data are taken from the same experiment as in a with a number of detected photons per emitter between 1800 and 6500 and a median photon value of 2800 d, 3D super resolved image of a 3 micrometers diameter sphere labelled with AF647 fluorescent probes conjugated to streptavidin, the capture range limits the observation to the bottom of the sphere close to the coverslip. e, Sphere xz cross section shows the geometric profile of the sphere. $f$, Comparison of the experimental heights of different single molecules detected and the theoretical heights calculated from the sphere geometrical model. The black curve represents the expected tendency and median values of ModLoc are represented by blue dots.

We performed complementary experiments using $40 \mathrm{~nm}$ diameter fluorescent nanobeads for which the emission level is controlled ${ }^{15,16,20}$ (see Supplementary Fig. 13). The localization precisions are also in very 
172 good agreement with CRLB calculations and the lateral and axial localization precisions are approximately 173 constant over the first seven microns in depth (with $3.4 \pm 1.2 \mathrm{~nm}$ and $7.6 \pm 2.1 \mathrm{~nm}$ ). Unlike most PSF 174 shaping methods, the lateral precision is not compromised on the axial one. In addition, modeling the 175 influence of photon noise per pixel resulting from an axial defocusing confirm the experimental 176 observations of a nearly constant localization precision of ModLoc. This is in sound contrast with 177 astigmatism performances which degrade rapidly with defocusing (Supplementary Fig. 14 and 15).

178 The accuracy, representing the discrepancy between the measured position and the real one, is another 179 important parameter in SMLM. This parameter is evaluated from images of $3 \mu \mathrm{m}$ diameter microspheres 180 labeled with Alexa Fluor 647 imaged in (d)STORM (Methods)(Fig. 2d) and their associated xz projection 181 (Fig. 2e). The measured axial positions of different localized fluorescent probes coincide with their 182 theoretical positions on the microsphere (Fig. 2f). These findings prove that ModLoc is a real asset for 3D 183 single molecule imaging especially for in depth imaging.

185 We now focus on ModLoc performances on biological structures observed in (d)STORM (Methods). COS7 186 cells are imaged in the first $600 \mathrm{~nm}$ close to the coverslip with a standard labeling of tubulin to visualize 187 their microtubule network (Fig. 3a-3c). The precision of ModLoc enables visualizing the microtubule 188 cylindrical structure (Fig. 3d). Direct visualization of their hollownesses is impeded by the labelling density 189 and the uncertainty in the microtubule orientations (Fig. 3e), however statistically accumulation over 190 several microtubule sections at various depths (see Fig. 3f and Supplementary Fig. 16) yields a radial 191 density profile which exhibits a central dip with a radius of $\sim 12.3 \mathrm{~nm}$ as expected from the microtubule 192 structure ${ }^{33,34}$ (Fig. 3g). The tail at higher radii originates from the distance distribution introduced by the 193 labelling. 

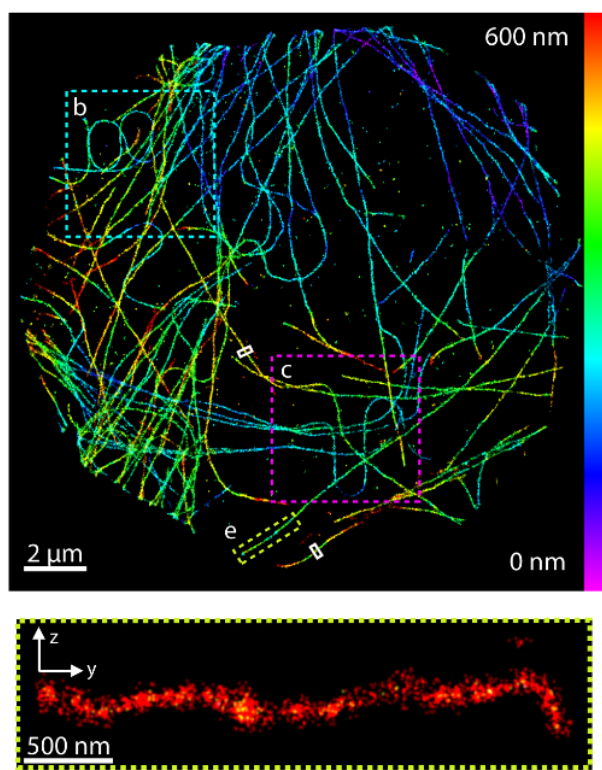

b

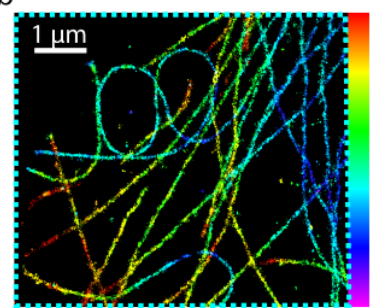

$f$

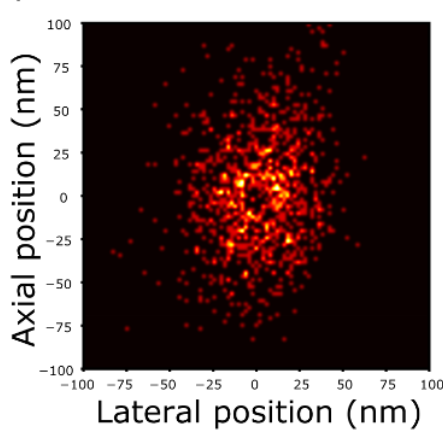

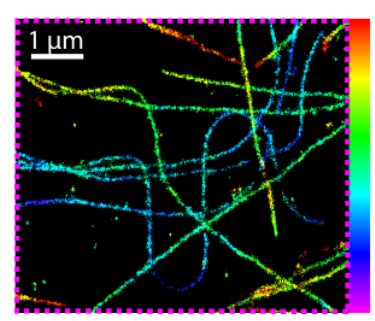

g

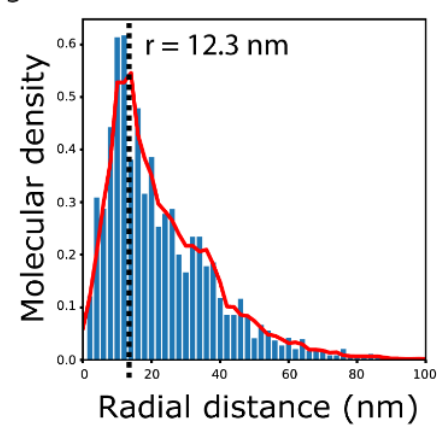

Figure 3 / 3D imaging of microtubules network in the first $600 \mathrm{~nm}$ in COS7 cell in (d)STORM. a- Full 3D imaging of tubulin in cos 7 cell at the coverslip labeled with AF 647 fluorescent probes, $\boldsymbol{b}$ zoom on the cyan ROI of $\boldsymbol{a}$. $\boldsymbol{c}$ zoom on the pink ROI in $\boldsymbol{a}$. $\boldsymbol{d}$ Different $y z$ cross sections highlighted by white ROI in $\boldsymbol{a}$, which evidenced the cylindrical structure microtubules. e zoom on the yellow ROI of $\boldsymbol{a}, y z$ cross section of a microtubule. $f$ Density map of detected fluorescent molecules for 7 different microtubules located at various depths within the capture range. gradial molecular density of $f$ exhibiting a central dip with a radius of $\sim 12.3 \mathrm{~nm}$.

We now study the performances of ModLoc at several micron depths in (d)STORM (Fig. 2f). Figure 4a shows clathrin pits repartition in COS7 cells labeled with AF 647 at a depth of 4 microns revealing the presence of the underlying nucleus. The yz cross sections of the clathrin pits (Fig. 4b) show morphologies similar to the one obtained near the coverslip (Fig. 4c) confirming the constant axial localization precision of ModLoc. Figures $4 \mathrm{~d}-4 \mathrm{~g}$ show mitochondria at 6 microns depth in COS7 cells. The uniformity of the localization precision is investigated by measurements of the thickness of mitochondria membranes at different depths (see Supplementary Figure 17). Results show that the measured thicknesses are unaffected by the axial position with a median value of $29.8 \pm 5.3 \mathrm{~nm}$. This value agrees with the reported values of mitochondria membranes $\sim 7.5 \mathrm{~nm}$ measured in cryotomography $\mathrm{y}^{35}$ ) and primary and secondary antibodies sizes $(\sim 20 \mathrm{~nm})$ as well as measurements with other super-resolution techniques ${ }^{36}$. 
a
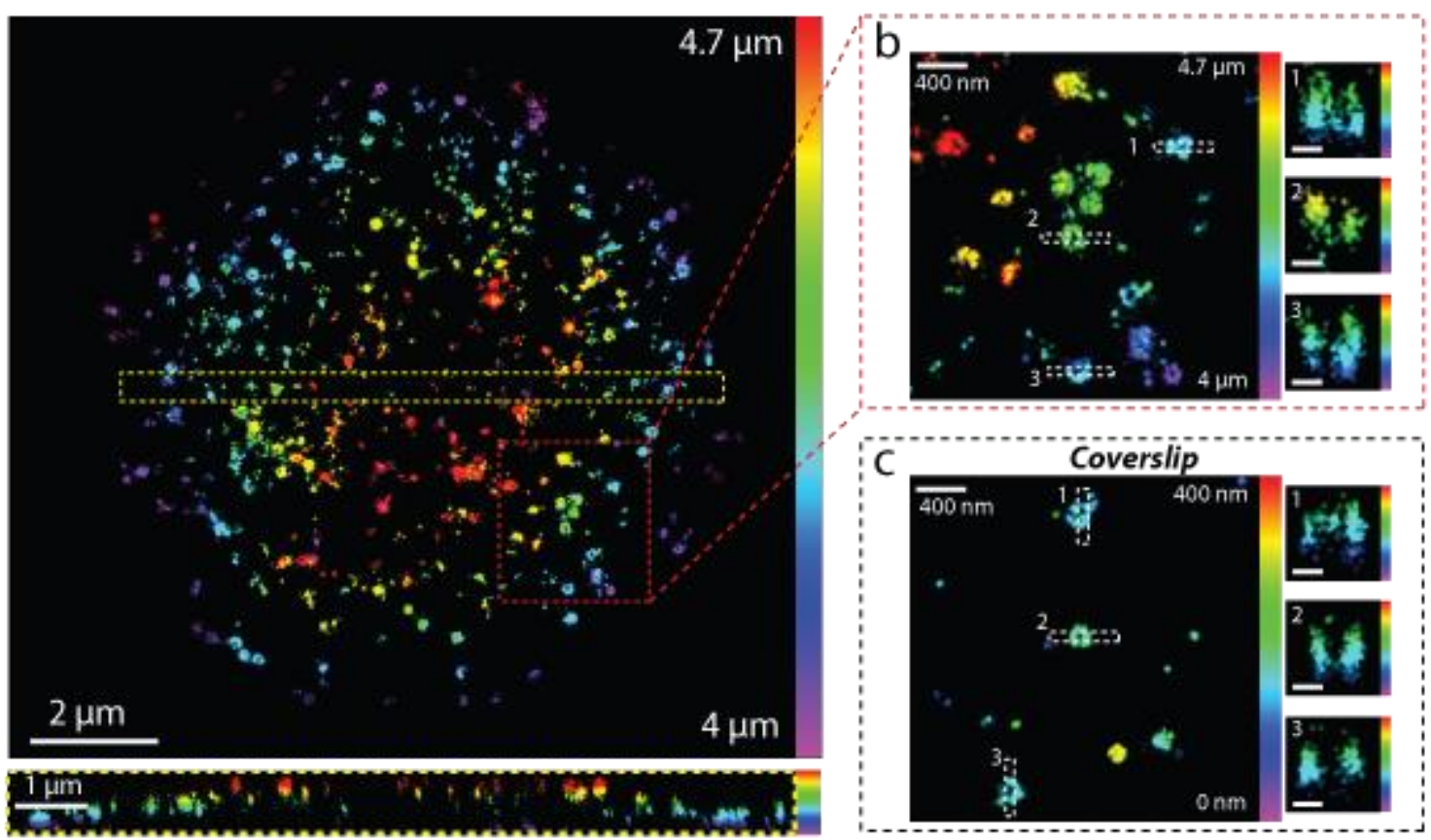

d
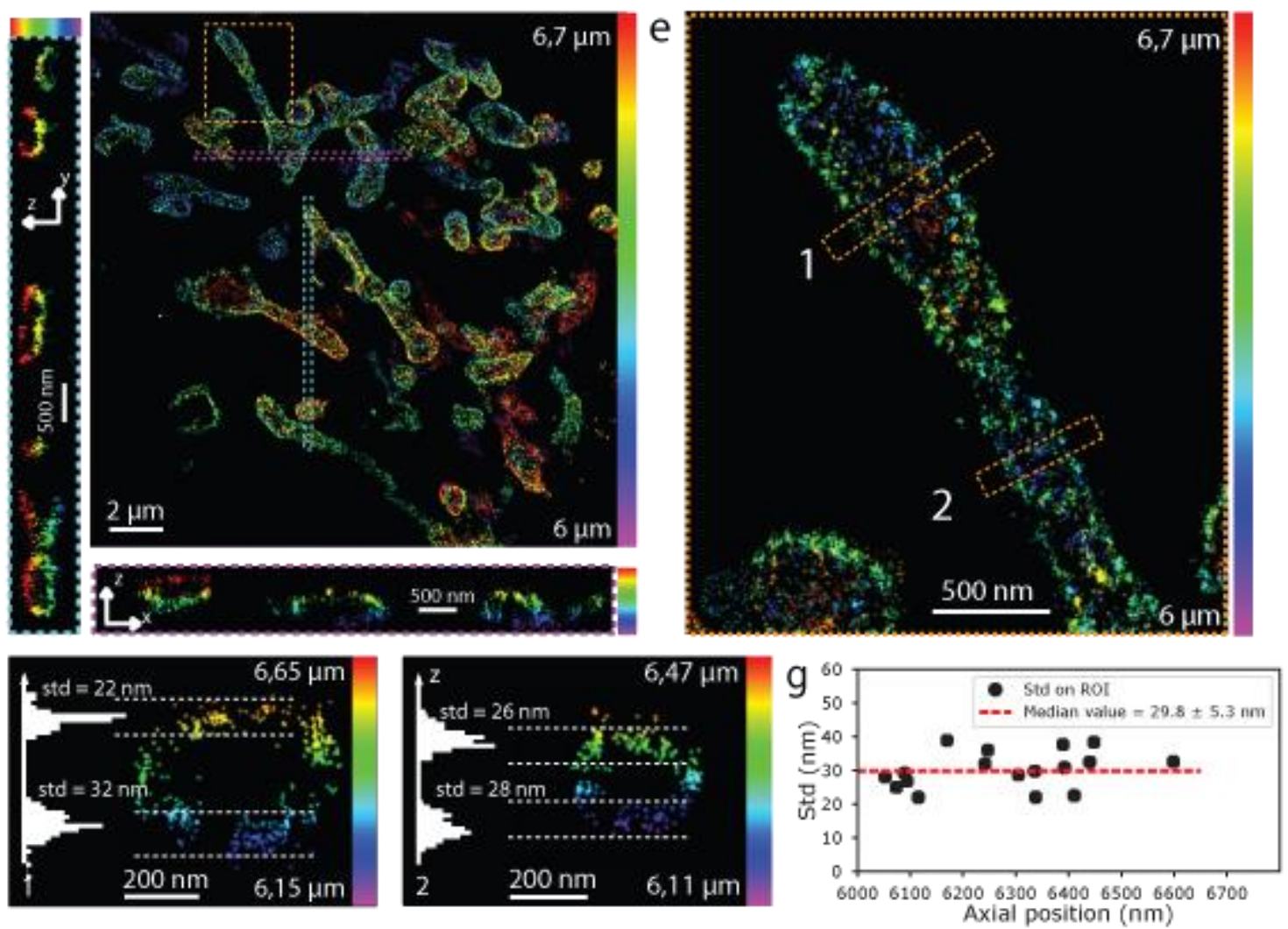

Figure 4 | 3D imaging of biological samples with ModLoc in (d)STORM. a, Top: full 3D imaging of clathrin in cos7 cell at 4 microns in depth with heavy chain labeled with AF 647 fluorescent probes. Bottom: xz cross section of the yellow ROI. The $z$ bar color is defined in the same interval as in the top. $\mathbf{b}$, Left: zoom on the red ROI of $\mathbf{a}$. Right: different $x z$ and $y z$ cross sections of the white ROI (scale $100 \mathrm{~nm}$ ). c, Left: 3D image of clathrin in cos7 at the coverslip with heavy chain labeled with AF 647 fluorescent probes. Right: different $x z$ and $y z$ cross sections of the white ROI (scale $100 \mathrm{~nm}$ ). d, 3D image of mitochondria in cos 7 cell at 6 microns in depth with Tom22 labelled with AF647, 2 cross sections of ROIs are shown at left and at the bottom of the full field image. The colorbar range is the same for the 3 images. e, Top: zoom on the orange ROI of $\mathrm{d}$. $\mathbf{f}$. XZ cross sections at different positions along one mitochondria (zone 1 and 2 in e), with axial histograms showing the membrane thickness at the top and the bottom of the mitochondria $\mathbf{g}$, Standard deviation of different histograms for membrane thickness measured at different depths showing uniform z precision over the whole capture range. 

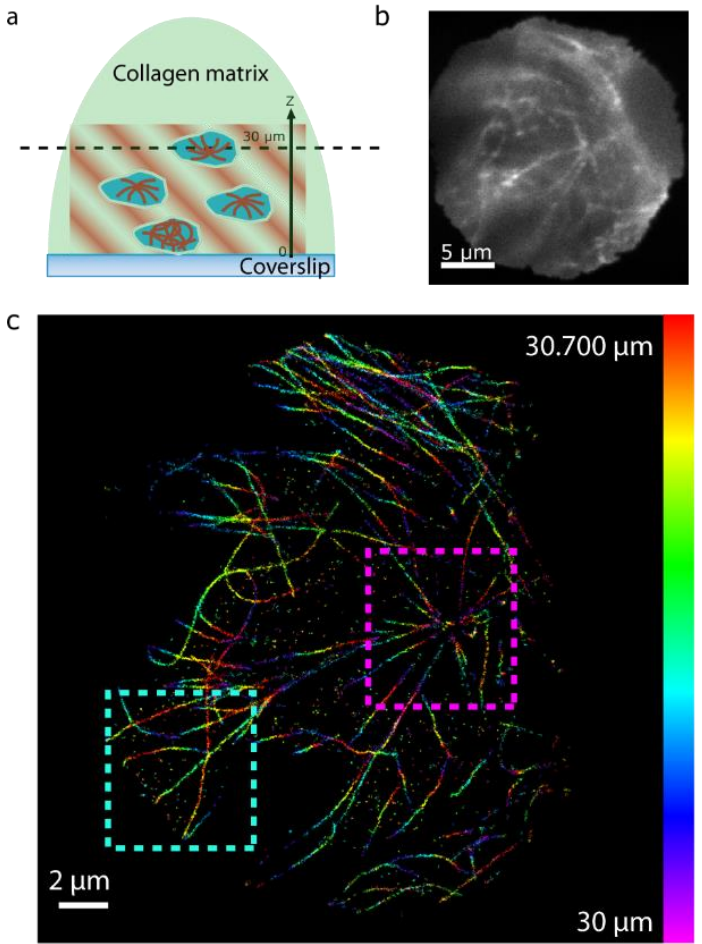

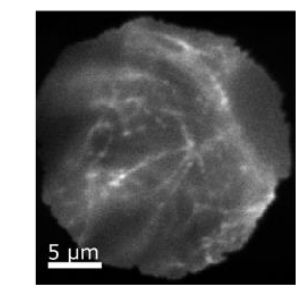

$30.700 \mu \mathrm{m}$

$30 \mu \mathrm{m}$
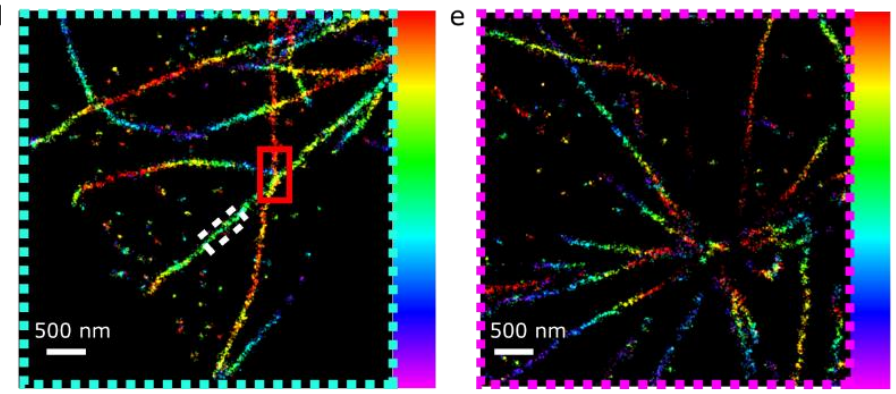

f Axial projection of 3 crossed microtubules

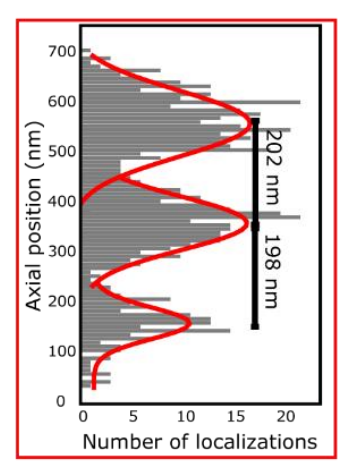

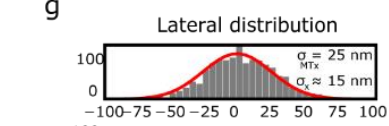

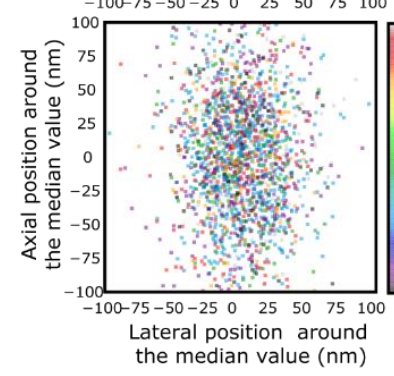

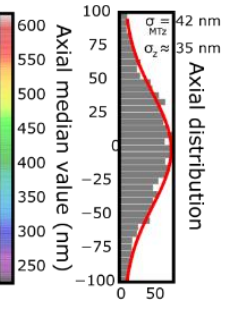

Figure 5 | 3D imaging of COS 7 cell microtubule grown in collagen matrix in(d)STORM . a, Schematic representation of COS 7 grown in a collagen matrix. b. Wide field image of $\cos 7$ cell tubulin at 30 microns in depth. c, 3D super resolved imaged of wide field represented in b. $\mathbf{d}$, Zoom on the blue ROI in c. e, Zoom on the purple ROI in c. f, Axial cross section of 3 microtubules (red $\mathrm{ROI}$ in c) at different depths in the capture range. g, Scatter plot represents projection of different microtubules (supplementary Figure 18 for ROI positions) along around $500 \mathrm{~nm}$ for each. The median axial position of each microtubule in encoded with the color bar and lateral and axial distribution are represented with histograms. Weighted standard deviation of each histogram $\sigma_{\mathrm{MTX}}$ $\sim 25 \mathrm{~nm}$ and $\sigma_{\mathrm{MTz}} \sim 42 \mathrm{~nm}$ gives an approximation of ModLoc localization precision $\sigma_{\mathrm{x}} \sim 15 \mathrm{~nm}$ and $\sigma_{\mathrm{z}} \sim 35 \mathrm{~nm}$ (Supplementary Figure 19).

Performances of ModLoc are evaluated under harsh conditions at even greater depths and in the presence

of strong optical aberrations. To perform observations at different depths, we use COS7 cells cultured in a

collagen matrix with standard labeling of tubulin to visualize their microtubule network (see Fig. 5a). The

wide-field image of the microtubule network at $30 \mu \mathrm{m}$ depth (taking into account the focal shift ${ }^{37}$ as the

microscope z-display indicated $50 \mu \mathrm{m}$ ) clearly shows the incidence of the aberrations and scattering

induced by the collagen matrix (Fig. 5b). The axial information can still be accurately retrieved at such large

depths as shown by the super-resolved 3D reconstructed image in Fig. 5c and the close-ups in Fig. 5d-5e. 
Manuscript NPHOT-2019-12-01587A-Z

247 Gaussian shape related to the radial fluorophore distribution profile around the microtubule (see Fig. 5g, 248 Supplementary Fig. 18 and 19). Measurements gives a lateral localization precision of about $15 \mathrm{~nm}$ and an 249 axial precision of around $35 \mathrm{~nm}$. The strong scattering properties of the collagen matrix impacts the 250 localization precision in all directions.

\section{Discussion}

252 ModLoc technique is easy to implement with a single objective configuration and offer a uniform axial 253 localization precision at various depths. It takes full advantage of the robustness of the excitation pattern 254 throughout the whole capture range compared to PSF-based techniques. ModLoc is well suited for deep biological samples imaging as demonstrated by images of the cytoskeleton and of various organelles up to $25630 \mu \mathrm{m}$.

ModLoc implementation could still be improved by modifying the excitation or the detection. The fringes pattern period could be reduced by using two counter propagating beams, either by using a reflecting mirror or in a 4Pi geometry which has the further advantage of doubling the collection efficiency ${ }^{16}$. In this case, the axial and lateral localizations become independent and an ultimate axial localization precision of $\sigma_{\text {ModLoc }} \approx 0.5 \sigma_{x}$ (see eq. 1 with $\alpha / 2=\theta=90^{\circ}$ ). The lock-in detection could be based alternatively on moving mirrors which, although limited in frequency and potential source of mechanical drift ${ }^{38}$, would be polarization independent and enhance the contrast.

iPALM such as ModLoc is based on a lock-in detection scheme to localize single emitters. While in iPALM the measured phase is that of the emitted electromagnetic field whose self-interference gives the position of the emitter, in ModLoc the phase is that of the amplitude modulation of the fluorescence emission which is related to the structured illumination pattern. The iPALM fundamental precision limit, like that of ModLoc, results from the shot noise of the molecular fluorescence signal and the spatial period of the modulation. With an equal spatial period of $\lambda / 2$ (with counter-propagating beam configuration) and the same number of collected photons, both techniques would approximately give the same localization precision. Creating an interference illumination pattern is however much less challenging then achieving 
273 imaging deeper in the sample restricted IPALM to thin samples and observations close to the coverslip,

274 deeper imaging was made possible only with adaptive optics ${ }^{19}$.

275 Both MINFLUX ${ }^{39}$ technique and ModLoc are based on a structured illumination to localize the molecule. In

276 the former case, the estimator and the illumination pattern in the shape of a doughnut have been

277 optimized to reach unmatched precision for a specific point, albeit breaking the translational invariance ${ }^{40}$.

278 ModLoc retains the translational invariance and is therefore designed to handle full field imaging.

279 ModLoc performances at depth shows that it is robust to optical aberrations. It mainly relies on the 280 robustness of the structured illumination obtained from the interference of two plane waves regarding 281 spherical aberrations. In the case of coherent illumination, the effect of aberrations is limited to a global 282 phase shift as shown in standard Structured Illumination Microscopy ${ }^{41,42}$. The phase detection of ModLoc 283 depends only on the relative number photons in each channel. Contrary to PSF engineering techniques, 284 this phase detection is independent of the PSF shape and thus robust to optical aberrations.

285 Taking advantage of the 3D imaging capability of ModLoc to decipher multiple proteins organization would 286 be a real asset. Several multiplexing strategies are fully compatible with ModLoc and could be readily 287 implemented, like sequential labeling approaches ${ }^{43,44}$ or by demixing red dyes with a dichroic beam288 splitter inserted at the detection ${ }^{45}$ or salvaging photons discarded by the dichroic ${ }^{46}$. Multiplexing strategy

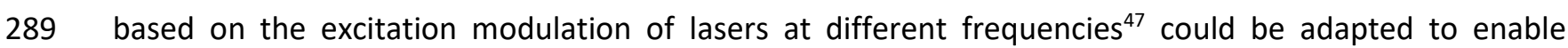
290 simultaneous multicolor imaging.

291 Standard amplitude modulation LIDAR and TOF cameras use an electronic driven modulation of the 292 excitation beam scattered by an object to obtain localization ${ }^{27}$. This technique couples spatial and 293 temporal modulations and limit the spatial periodicity of the excitation to typically a few meters. The 294 ModLoc structured illumination by interferences and its independent temporal shift enables a decoupling 295 of the spatial and temporal frequencies and a spatial period down to the micrometer range. This could 296 find potential applications in LIDAR and TOF camera to reach really high localization precision 297 (Supplementary Fig. 20). 
Manuscript NPHOT-2019-12-01587A-Z

The generic concept of ModLoc based on lock-in detection is well suited to perform fast multiple frequency demodulation without compromising on the acquisition time nor discarding ON-time emitters, should it be for multiple direction localization or for additional information at the single molecule level, such as the molecular orientation or the local chemical environment through the fluorescence lifetime measurement.

\section{Methods}

Optical setup. A complete schematic of the optical setup used is presented in Figure 1a. We used a Nikon Eclipse Ti inverted microscope with a Nikon Perfect Focus System. The dichroic and filters in the microscope cube are from Semrock (Di03-R635-t1-25x36 and BLP01-635R-25). The excitation was performed using a 639-nm laser (Genesis MX 639, 1W, Coherent), couple to a single-mode optical fiber maintaining polarization. The laser beam was separated in 2 paths with a 50/50 cube beamsplitter (BS010, Thorlabs) and recombined in the sample through a Nikon APO TIRF x60 1.49 NA oil immersion objective lens. The fluorescence was collected through the same objective lens, sent in the demodulation module and recorded on four quadrants of a 512×512-pixel EMCCD camera (iXon3, Andor). The camera was placed at the focal plane of the module of magnification 2.6 in order to get an optical pixel size of $100 \mathrm{~nm}$. The phase modulation at the excitation is performed by an electro optical modulator (EOM) (EO-PM-NR-C1, Thorlabs), while the phase sorting in the detection path is performed by a Pockels cell (CF1043-20SG$500 / 700$, Fast pulse) controlled by a high voltage power amplifier (10/10B-HS, TREK). The synchronization was provided thanks to a 4-channel generator that triggers the EOM, the Pockels cell and the camera.

Experimental demodulation process. Sampling of the temporal modulated intensity is performed by a homemade demodulation module composed of polarizing components and an active Pockels cell placed before the camera. The module creates 4 different channels accessible to the fluorescent signal and each channel is acquired in a subarray on the camera. The state change of the Pockels cell allow to modify the active channel of the fluorescence intensity and is synchronized with the excitation pattern position in order to collect the fluorescence of in each subarray. The fluorescence transmission for each state of the Pockels cell is described in Supplementary Note 1 and Supplementary Figure 5. 
324 Calibration samples preparation. To obtain the localization precisions displayed in Supplementary figure 32513 , we prepared a sample of 40-nm diameter dark red fluorescent nanobeads (10720, Thermo Fisher) 326 randomly distributed on fixed and non-labeled COS7 cells (fixation with 4\%PFA). This operation allows us 327 to obtain a sparse sample with isolated fluorescent nanobeads at different randomly heights. This sample 328 also allows us to be closer to experimental conditions of 3D single molecule imaging. We added $25 \mu \mathrm{L}$ of a $32910^{-5}$ diluted stock solution in $1 \mathrm{ml}$ of Phosphate Buffer Saline (PBS) and we wait 15 minutes before 330 observation for the beads to deposit.

331 The calibration of the tilt angle described Supplementary Note 1 and Supplementary Figure 10 was 332 performed by using coated biotin microspheres (Kisker Biotech, PC-B-3.0) labeled with AF647-steptravidin conjugated fluorophores ${ }^{48}$. We prepared a solution containing $500 \mu \mathrm{L}$ of water, $500 \mu \mathrm{L}$ of PBS, $30 \mu \mathrm{L}$ of stock microspheres solution and $1 \mu \mathrm{L}$ of streptavidin-functionalized AF647 (Life Technologies, S21374). The solution was centrifuged during 30 minutes at $10 \mathrm{krpm}$ and the liquid phase was removed and replaced with $100 \mu \mathrm{L}$ of PBS. Then, we vortexed the solution in order to dissolved the deposit. We added $1 \mathrm{ml}$ of 337 PBS to $50 \mu \mathrm{L}$ of the final solution on the coverslip and we waited 1 hour before starting the acquisitions.

The influence of the modulation frequency on the precision of single molecule events (Supplementary figure 4), was evaluated following the previous protocol adapted for DNA-PAINT imaging. Streptavidin coated microspheres (Spherotech SVP60-5) were conjugated with biotin DNA strand (BiotinTTATACATCTA, Metabion). A solution containing $500 \mu \mathrm{L}$ of water, $500 \mu \mathrm{L}$ of PBS, $30 \mu \mathrm{L}$ of stock microspheres solution and $1 \mu \mathrm{L}$ of biotin-DNA Strand $(100 \mu \mathrm{M})$ was centrifuged during 30 minutes at 10 krpm and the liquid phase was removed and replaced with $100 \mu \mathrm{L}$ of buffer (Imaging buffer, Massive Photonics). We added $1 \mathrm{ml}$ of buffer to $50 \mu \mathrm{L}$ of the final solution on the coverslip and we waited 1 hour before starting the acquisitions. $0.4 \mathrm{nM}$ of DNA-Strand imager labelled with ATTO647N was added to the solution (CTAGATGTAT-ATTO647N Metabion).

Pattern excitation was calibrated by imaging single fluorescent fluorophores at the coverslip. The sample is obtained by diluting $1 \mu \mathrm{L}$ of stock AF647 florescent probe solution in $500 \mu \mathrm{L}$ of dSTORM buffer (Abbelight dSTORM buffer). Calibration process are developed in Supplementary Note 1 et Supplementary Fig. 10. 


\section{Biological samples preparation.}

351 Cell culture

352 COS-7 cells were grown in DMEM with 10\% FBS, 1\% L-glutamin and 1\% penicillin/streptomycin (Life 353 Technologies) at $37^{\circ} \mathrm{C}$ and $5 \% \mathrm{CO} 2$ in a cell culture incubator. For U2OS culture, DMEM was replaced by 354 McCoy's 5A medium. Several days later, they could be plated at low confluence on cleaned round $25 \mathrm{~mm}$ 355 diameter high resolution\# 1.5 glass coverslips (Marienfield, VWR).

357 After 24 hours, the cells were washed three times with PHEM solution (60 mM PIPES, 25 mM HEPES, 5 mM 358 EGTA and $2 \mathrm{mM} \mathrm{Mg}$ acetate adjusted to $\mathrm{pH} 6.9$ with $1 \mathrm{M} \mathrm{KOH}$ ) and fixed for $20 \mathrm{~min}$ in $4 \%$ PFA, $0.02 \%$ 359 glutaraldehyde and 0.5\% Triton. They were then washed 3 times in PBS (Invitrogen, 003000). Up to this 360 fixation step, all chemical reagents were pre-warmed at $37^{\circ} \mathrm{C}$. The cells were permeabilized 10 min with 361 PBS $+0.1 \%$ Triton $\mathrm{X}-100$ before to be reduced 10 min with $\mathrm{NaBH} 4$.

\section{Cell fixation for Clathrin and Mitochondria imaging}

363 The cells were washed three times with PHEM solution $60 \mathrm{mM}$ PIPES, 25 mM HEPES, 5 mM EGTA and 2 $364 \mathrm{mM} \mathrm{Mg}$ acetate adjusted to $\mathrm{pH} 6.9$ with $1 \mathrm{M} \mathrm{KOH}$ ) and fixed for $10 \mathrm{~min}$ in $4 \%$ PFA. They were then washed 3653 times in PBS (Invitrogen, 003000). Up to this fixation step, all chemical reagents were pre-warmed at $36637^{\circ} \mathrm{C}$. The cells were reduced $7 \mathrm{~min}$ with $50 \mathrm{mM} \mathrm{NH} 4 \mathrm{Cl}$.

U2OS Cell labelling for DNA-PAINT imaging

368 Saturation step was performed by washing in PBS three times before being blocked 30 min in PBS $+3 \%$ $369 \mathrm{BSA}+0.5 \%$ Triton. The cells were incubated 1 hour at $37^{\circ} \mathrm{C}$ with 1:300 mouse anti-Clathrin Heavy Chain 370 antibody (Sigma Aldrich, C1860) in PBS $+1 \%$ BSA. This was followed by three washing steps in PBS $+3 \%$ $371 \mathrm{BSA}$, incubation during $1 \mathrm{~h}$ at $37^{\circ} \mathrm{C}$ with 1:300 anti-mouse combined to DNA-PAINT strand (Massive Photonics) diluted in antibody buffer (Massive Photonics). Three more washes with washing buffer 
Manuscript NPHOT-2019-12-01587A-Z

373 (Massive Photonics) was performed before imaging with a concentration of $0.2 \mathrm{~nm}$ of DNA-Strand imager 374 labelled with ATTO647N (CTAGATGTAT-ATTO647N Metabion). No post-fixation was performed COS7 Cell labeling for tubulin imaging in dSTORM

Saturation step was performed by washing in PBS three times before being blocked 15 min in PBS + 1\% BSA. The cells were incubated 1 hour at $37^{\circ} \mathrm{C}$ with 1:300 mouse anti- $\alpha$-tubulin antibody (Sigma Aldrich, T6199) in PBS $+1 \%$ BSA. This was followed by three washing steps in PBS $+1 \%$ BSA, incubation during 45 min at $37^{\circ} \mathrm{C}$ with 1:300 goat anti-mouse AF647 antibody (Life Technologies, A21237) diluted in PBS 1\% BSA and three more washes in PBS. A post-fixation step was performed using PBS with $3.6 \%$ formaldehyde during $15 \mathrm{~min}$. The cells were washed in PBS three times and then reduced during 10 min with $50 \mathrm{mM}$ $\mathrm{NH} 4 \mathrm{Cl}$ (Sigma Aldrich, 254134), followed by three additional washes in PBS. $\mathrm{BSA}+0.5 \%$ Triton. The cells were incubated 1 hour at $37^{\circ} \mathrm{C}$ with $1: 300$ mouse anti-Clathrin Heavy Chain antibody (Sigma Aldrich, C1860) in PBS $+3 \%$ BSA. This was followed by three washing steps in PBS $+3 \%$ BSA, incubation during $45 \mathrm{~min}$ at $37^{\circ} \mathrm{C}$ with 1:300 goat anti-mouse AF647 antibody (Life Technologies, A21237) diluted in PBS 1\% BSA and three more washes in PBS. A post-fixation step was performed using PBS with 3.6\% formaldehyde during 15 min. The cells were washed in PBS three times and then reduced during 10 min with $50 \mathrm{mM} \mathrm{NH4Cl}$ (Sigma Aldrich, 254134), followed by three additional washes in PBS. $\mathrm{BSA}+0.5 \%$ Triton. The cells were incubated 1 hour at $37^{\circ} \mathrm{C}$ with 1:300 mouse anti-TOM22 antibody (Sigma Aldrich, T6319) in PBS + 3\% BSA. This was followed by three washing steps in PBS +3\% BSA, incubation during 45 min at $37^{\circ} \mathrm{C}$ with 1:300 goat anti-mouse AF647 antibody (Life Technologies, A21237) diluted in 
formaldehyde during $15 \mathrm{~min}$. The cells were washed in PBS three times and then reduced during $10 \mathrm{~min}$ with $50 \mathrm{mM} \mathrm{NH} 4 \mathrm{Cl}$ (Sigma Aldrich, 254134), followed by three additional washes in PBS.

COS-7 cells were grown in DMEM with $10 \%$ FBS, 1\% L-glutamin and 1\% penicillin/streptomycin (Life Technologies) at $37^{\circ} \mathrm{C}$ and $5 \% \mathrm{CO} 2$ in a cell culture incubator. Upon reaching confluency, the cells in DMEM were added to $80 \%$ collagen, $10 \%$ MEM and $5.83 \%$ neutralizing solution (RAFT3D Cell Culture Kit, LONZA). The last step was done on ice at $4^{\circ} \mathrm{C}$. Then, the cells were plated at $2,510^{-5}$ cells per well on cleaned round $25 \mathrm{~mm}$ diameter high resolution \#1.5 glass coverslips (Marienfield, VWR). After $30 \mathrm{~min}$ at $37^{\circ} \mathrm{C}$ in the incubator, the exceeding solution was removed and replaced by DMEM without phenol red with $10 \%$ FBS, 1\% L-glutamin and 1\% penicillin/streptomycin. After 24 hours, we did the same steps as described 407 previously.

Imaging modalities. Biological experiments were performed mainly in (d)STORM conditions with a classical dSTORM buffer (Smart Kit Abbelight), with a typical number of image acquisition between 10000 to 40000 images and an excitation of $2 \mathrm{~kW} . \mathrm{cm}^{2}$. Results presented in Figure $2 \mathrm{a}, 2 \mathrm{~b}$ and $2 \mathrm{c}$ have been obtained in DNA-PAINT, with a similar number of acquisition but an excitation power reduced to $0.4 \mathrm{~kW} . \mathrm{cm}^{2}$

Cramèr-Rao Lower Bounds. Calculation are developed in Supplementary Note 1. The CRLB of ModLoc is obtained from the classical CRLB of the lateral detection and the CRLB of the phase. Influence of the number of photons and the background noise are represented in Supplementary Figure 11 and

415 Supplementary Figure 12. Comparison with astigmatism method are illustrated in Supplementary Figure 41614 and Supplementary Figure 15.

417 Data processing wokflow. All processing scripts are based on public available software.

419 First, we remove the background signal on the 512x512-pixel raw frames by subtracting the temporal 420 median of the 10 previous and 10 next frames, pixel per pixel. Then, we detect the PSFs by using a wavelet 421 filtering ${ }^{49}$ associated to a low intensity threshold and large spot width filtering parameters. This is 
422

necessary due to the large disparities in fluorescence intensity obtained by modulation in the frame. We measure the nanometric position by Gaussian fitting using the Gpufit maximum likelihood estimate Python toolkit ${ }^{50}$. The intensity is then obtained by a photon counting over a $1 \mu \mathrm{m} \times 1 \mu \mathrm{m}$ around the center of the point spread function.

The second step recombines the four intensities to obtain the phase information for each molecule. The localization array is split in four parts corresponding to the four detection channels. Lateral positions detected in other channels by Gaussian fitting are corrected by using affine transformations in order to correct magnification, rotation and translation caused by misalignment of the detection paths. The top left channel is used as a reference for this operation, and affine transformation parameters are obtained with a simple registration algorithm (see supplementary fig. 6 for an estimation of registration errors). We then merge the four localizations corresponding to each fluorophore and we apply transmission correction coefficients on each intensity channel determined for the considered fluorescent probe. Transmission coefficient are obtained by acquiring fluorescence in each channel without any modulation of the intensity, at the beginning of the experiment. Finally, we calculate the phase from the four intensities for each molecule. The axial position is obtained by using the $x$ coordinates from the Gaussian fitting and the phase measurements as:

\section{Drift correction}

Drifts are corrected using an axial and lateral custom-made program based on a Direct Cross Correlation (DCC) algorithm ${ }^{32}$. The localization list is divided into several temporal slices based on the frame numbers, the reference slice being chosen as the first and the lateral drift correction is performed at first. The transverse temporal drift is obtained by a $2 \mathrm{D}$ cross-correlation of 2 temporal stack. Thus, it is possible to obtain a temporal drift curve whose sampling depends of the size of temporal Stack. When lateral drifts are corrected, the operation is repeated along the axial direction. The localizations are binned in 3D images 
446 with a voxel size typically set to $60 \times 60 \times 40 \mathrm{~nm}$ in $x, y, z$, and the slice size is generally 500 frames. This

447 operation can be repeated several times by changing the slice size in order to refine the precision.

448 Localization precision estimation from repeated localizations on single emitters

449 Localization precision plotted in figure 2 a-c were obtained by calculating the standard deviation on the 450 positions of localized molecules on successive frames. Molecules present on a radius less than $30 \mathrm{~nm}$ are 451 considered to be identical. To be considered, the molecule must be active on 4 successive frames at the 452 minimum and 10 successive frames at the maximum. The positions obtained on the first and last image 453 are not considered for the standard deviation calculations.

Data availability

Data are available upon reasonable request to the corresponding author.

456 Code avaibility

457 Processing code are based on already published solutions as described in the supplementary

$458 \quad$ References

459 1. Betzig, E. et al. Imaging Intracellular Fluorescent Proteins at Nanometer Resolution. Science $\mathbf{3 1 3 ,}$ $460 \quad 1642-1645$ (2006).

461 2. Hess, S. T., Girirajan, T. P. K. \& Mason, M. D. Ultra-High Resolution Imaging by Fluorescence 462 Photoactivation Localization Microscopy. Biophysical Journal 91, 4258-4272 (2006).

463 3. Rust, M. J., Bates, M. \& Zhuang, X. Sub-diffraction-limit imaging by stochastic optical reconstruction 464 microscopy (STORM). Nat Methods 3, 793-796 (2006).

465 4. Heilemann, M. et al. Subdiffraction-Resolution Fluorescence Imaging with Conventional Fluorescent 466 Probes. Angewandte Chemie International Edition 47, 6172-6176 (2008).

467 5. von Diezmann, A., Shechtman, Y. \& Moerner, W. E. Three-Dimensional Localization of Single 468 Molecules for Super-Resolution Imaging and Single-Particle Tracking. Chemical Reviews 117, 72447275 (2017). 
Manuscript NPHOT-2019-12-01587A-Z

470

6. Ram, S., Prabhat, P., Chao, J., Ward, E. S. \& Ober, R. J. High Accuracy 3D Quantum Dot Tracking with Multifocal Plane Microscopy for the Study of Fast Intracellular Dynamics in Live Cells. Biophysical Journal 95, 6025-6043 (2008).

7. Juette, M. F. et al. Three-dimensional sub-100 $\mathrm{nm}$ resolution fluorescence microscopy of thick samples. Nature methods 5, 527-9 (2008).

8. Hajj, B., El Beheiry, M., Izeddin, I., Darzacq, X. \& Dahan, M. Accessing the third dimension in localization-based super-resolution microscopy. Phys. Chem. Chem. Phys. 16, 16340-16348 (2014).

9. Huang, B., Wang, W., Bates, M. \& Zhuang, X. Three-Dimensional Super-Resolution Imaging by Stochastic Optical Reconstruction Microscopy. Science 319, 810-813 (2008).

10. Pavani, S. R. P. et al. Three-dimensional, single-molecule fluorescence imaging beyond the diffraction limit by using a double-helix point spread function. Proceedings of the National Academy of Sciences 106, 2995-2999 (2009).

11. Shechtman, Y., Weiss, L. E., Backer, A. S., Sahl, S. J. \& Moerner, W. E. Precise Three-Dimensional Scan-Free Multiple-Particle Tracking over Large Axial Ranges with Tetrapod Point Spread Functions. Nano Letters 15, 4194-4199 (2015).

12. Badieirostami, M., Lew, M. D., Thompson, M. A. \& Moerner, W. E. Three-dimensional localization precision of the double-helix point spread function versus astigmatism and biplane. Appl. Phys. Lett. 97, 161103 (2010).

13. Bourg, N. et al. Direct optical nanoscopy with axially localized detection. Nature Photonics 9, 587593 (2015).

14. Deschamps, J., Mund, M. \& Ries, J. 3D superresolution microscopy by supercritical angle detection. 11 (2014).

15. Cabriel, C. et al. Combining 3D single molecule localization strategies for reproducible bioimaging. Nat Commun 10, 1-10 (2019).

16. Shtengel, G. et al. Interferometric fluorescent super-resolution microscopy resolves 3D cellular ultrastructure. Proceedings of the National Academy of Sciences of the United States of America 106, 3125-3130 (2009). 
17. Wang, G., Hauver, J., Thomas, Z., Darst, S. A. \& Pertsinidis, A. Single-Molecule Real-Time 3D Imaging of the Transcription Cycle by Modulation Interferometry. Cell 167, 1839-1852.e21 (2016).

18. Aquino, D. et al. Two-color nanoscopy of three-dimensional volumes by 4Pi detection of stochastically switched fluorophores. Nature Methods 8, 353-359 (2011).

19. Huang, F. et al. Ultra-High Resolution 3D Imaging of Whole Cells. Cell 166, 1028-1040 (2016).

20. Bon, P. et al. Self-interference 3D super-resolution microscopy for deep tissue investigations. Nature Methods 15, 449 (2018).

21. von Diezmann, A., Shechtman, Y. \& Moerner, W. E. Three-Dimensional Localization of Single Molecules for Super-Resolution Imaging and Single-Particle Tracking. Chem. Rev. 117, 7244-7275 (2017).

22. Burke, D., Patton, B., Huang, F., Bewersdorf, J. \& Booth, M. J. Adaptive optics correction of specimen-induced aberrations in single-molecule switching microscopy. Optica 2, 177 (2015).

23. LIGO Scientific Collaboration and Virgo Collaboration et al. Observation of Gravitational Waves from a Binary Black Hole Merger. Phys. Rev. Lett. 116, 061102 (2016).

24. Zernike, F. How I Discovered Phase Contrast. Science 121, 345-349 (1955).

25. Beaurepaire, E., Boccara, A. C., Lebec, M., Blanchot, L. \& Saint-Jalmes, H. Full-field optical coherence microscopy. Opt. Lett., OL 23, 244-246 (1998).

26. Taylor, R. W. et al. Interferometric scattering microscopy reveals microsecond nanoscopic protein motion on a live cell membrane. Nature Photonics 13, 480-487 (2019).

27. TOF Range-Imaging Cameras. (Springer-Verlag, 2013).

28. Cappello, G. et al. Myosin V stepping mechanism. Proc Natl Acad Sci U S A 104, 15328-15333 (2007).

29. Busoni, L., Dornier, A., Viovy, J.-L., Prost, J. \& Cappello, G. Fast subnanometer particle localization by traveling-wave tracking. Journal of Applied Physics 98, 064302 (2005).

30. Reymond, L. et al. SIMPLE: Structured illumination based point localization estimator with enhanced precision. Opt. Express, OE 27, 24578-24590 (2019).

31. Cnossen, J. et al. Localization microscopy at doubled precision with patterned illumination. bioRxiv 554337 (2019) doi:10.1101/554337. 
Manuscript NPHOT-2019-12-01587A-Z

524

32. Wang, Y. et al. Localization events-based sample drift correction for localization microscopy with redundant cross-correlation algorithm. Optics Express 22, 15982 (2014).

33. Weber, K., Rathke, P. C. \& Osborn, M. Cytoplasmic microtubular images in glutaraldehyde-fixed tissue culture cells by electron microscopy and by immunofluorescence microscopy. Proceedings of the National Academy of Sciences 75, 1820-1824 (1978).

34. Zwettler, F. U. et al. Molecular resolution imaging by post-labeling expansion single-molecule localization microscopy (Ex-SMLM). Nature Communications 11, (2020).

35. Gold, V. A. M. et al. Visualizing active membrane protein complexes by electron cryotomography. Nature Communications 5, 4129 (2014).

36. Xu, F. et al. Three-dimensional nanoscopy of whole cells and tissues with in situ point spread function retrieval. Nature Methods 17, 531-540 (2020).

37. Bratton, B. P. \& Shaevitz, J. W. Simple Experimental Methods for Determining the Apparent Focal Shift in a Microscope System. PLOS ONE 10, e0134616 (2015).

38. Gu, L. et al. Molecular resolution imaging by repetitive optical selective exposure. Nat Methods 1-5 (2019) doi:10.1038/s41592-019-0544-2.

39. Balzarotti, F. et al. Nanometer resolution imaging and tracking of fluorescent molecules with minimal photon fluxes. Science 355, 606-612 (2017).

40. Gwosch, K. C. et al. MINFLUX nanoscopy delivers multicolor nanometer 3D-resolution in (living) cells. bioRxiv 734251 (2019) doi:10.1101/734251.

41. Arigovindan, M., Sedat, J. W. \& Agard, D. A. Effect of depth dependent spherical aberrations in 3D structured illumination microscopy. Opt. Express, OE 20, 6527-6541 (2012).

42. Booth, M., Andrade, D., Burke, D., Patton, B. \& Zurauskas, M. Aberrations and adaptive optics in super-resolution microscopy. Microscopy (Oxf) 64, 251-261 (2015).

43. Jungmann, R. et al. Multiplexed 3D cellular super-resolution imaging with DNA-PAINT and ExchangePAINT. Nature Methods 11, 313-318 (2014).

44. Klevanski, M. et al. Automated highly multiplexed super-resolution imaging of protein nanoarchitecture in cells and tissues. Nat Commun 11, 1-11 (2020). 
Manuscript NPHOT-2019-12-01587A-Z

551 45. Lampe, A., Haucke, V., Sigrist, S. J., Heilemann, M. \& Schmoranzer, J. Multi-colour direct STORM with red emitting carbocyanines. Biology of the Cell 104, 229-237 (2012).

46. Zhang, Y. et al. Nanoscale subcellular architecture revealed by multicolor three-dimensional salvaged fluorescence imaging. Nat Methods 17, 225-231 (2020).

47. Gómez-García, P. A., Garbacik, E. T., Otterstrom, J. J., Garcia-Parajo, M. F. \& Lakadamyali, M. Excitation-multiplexed multicolor superresolution imaging with fm-STORM and fm-DNA-PAINT. PNAS 115, 12991-12996 (2018).

48. Cabriel, C., Bourg, N., Dupuis, G. \& Lévêque-Fort, S. Aberration-accounting calibration for 3D singlemolecule localization microscopy. Opt. Lett., OL 43, 174-177 (2018).

49. Izeddin, I. et al. Wavelet analysis for single molecule localization microscopy. Optics Express 20, 2081 (2012).

50. Przybylski, A., Thiel, B., Keller-Findeisen, J., Stock, B. \& Bates, M. Gpufit: An open-source toolkit for GPU-accelerated curve fitting. Sci Rep 7, 1-9 (2017).

\section{Acknowledgements}

P.J. acknowledge a master funding form GDR ImaBio, and PhD funding from IDEX Paris Saclay (ANR-11IDEX-0003-02). M.B. is funded by the Labex PALM. We acknowledge the contribution of the Centre de Photonique BioMédicale to cell culture and labeling. We also thank Guillaume Dupuis for discussion and Surabhi Sreenivas for a careful reading of the paper. We thank Abbelight for the free use of NEMO software and dSTORM buffers. This work was supported by the AXA research fund, the ANR (LABEX WIFI, ANR-10LABX-24), ANR MSM-Modulated super-resolution microscopy (ANR-17-CE09-0040), the valorization program of the IDEX Paris Saclay and of Labex PALM.

\section{Author contributions} acquisitions, CRLB calculations and data analysis. P.J. and E.F. carried out simulations. N.B. developed the 
Manuscript NPHOT-2019-12-01587A-Z

577 (d)STORM buffer. N.B., C.C. and P.J. optimized the immunofluorescence protocol. P.J., C.C. and S.L.F

578 prepared the COS-7 and U2OS cells samples. M.B designed the 3D sample protocol. All authors 579 contributed to writing the manuscript.

\section{Competing financial interests}

581 The CNRS has deposited a patent FR3054321-A1 on the 25 July 2016 to protect this work, currently

582 under international extension. S.L.F, E.F. and N.B. are co-inventors. 